\title{
Surface Structure and Analysis
}

With Scanning Tunneling Microscopy

and Electron Tunneling Spectroscopy

PROGRESS REPORT

May 1, 1991 - April 30, 1992

\section{GRANT NO: DE-FGO5-89ER45072}

\author{
Robert V. Coleman \\ Department of Physics \\ University of Virginia \\ Charlottesville, $\mathrm{A}$
}

\section{PREPARED FOR THE DEPARTMENT OF ENERGY \\ UNDER GRANT NO: DE-FGO5-89ER45072}

\section{DISCLAIMER}

This report was prepared as an account of work sponsored by an agency of the United States Government. Neither the United States Government nor any agency thereof, nor any of their employees, makes any warranty, express or implied, or assumes any legal liability or responsibility for the accuracy, completeness, or usefulness of any information, apparatus, product, or process disclosed, or represents that its use would not infringe privately owned rights. Reference herein to any specific commercial product, process, or service by trade name, trademark, manufacturer, or otherwise does not necessarily constitute or imply its endorsement, recommendation, or favoring by the United States Government or any agency thereof. The views and opinions of authors expressed herein do not necessarily state or reflect those of the United States Government or any agency thereof. 


\section{Publications}

A. Reprints of Articles Published During Current Grant Year $*$ (Rernoved)

1. "Atomic Force Microscopy and Scanning Tunneling Microscopy of Charge-Density Waves in IT-TaSe ${ }_{2}$ ", Phys. Rev. $\underline{\text { B }}$, 9255 (1990). (DOE-ER45072-40).

2. "Spectroscopy of Dichalcogenides and Trichalcogenides Using Scanning Tunneling Microscopy", Journ. Vac. Science and Technology , 9, 1048 (1991).

3. "Long Range Modulation of the Charge-Density Wave Structure in IT- $\mathrm{TaS}_{2}$ Studied by Scanning Tunneling Microscopy", Journ. Vac. Science and Technology, 2, 490 (1991), (DOE-ER45072-42).

4. "Atomic Force Microscopy of Charge-Density Waves and Atoms on IT-TaSe ${ }_{2}$, IT$\mathrm{TaS}_{2}, \mathrm{IT}-\mathrm{TiSe}_{2}$ and $2 \mathrm{H}-\mathrm{NbSe}_{2}$ ", Journ. Vac. Science and Technology, 2, 1032 (1991), (DOE-ER45072-43).

5. "Charge-Density Wave Structure in $\mathrm{NbSe}_{3}$ Determined by Scanning Tunneling Microscopy", Phys. Rev. Lett. 66, 1318 (1991), (DOE-ER45072-44).

B. Publications Prepared During Current Grant Period

1. "Amplitude Modulation of Charge-Density-Wave Domains in IT-TaS ${ }_{2}$ at $300 \mathrm{~K}$ ", R.V. Coleman, W.W. McNairy, and C.G. Slough, Phys. Rev. B, to be published, (DOEER45072-46).

2. "STM and AFM of Layer Structure Crystals", R.V. Coleman, Zhenxi Dai, W.W. McNairy, C.G. Slough, and Chen Wang" to be published as a chapter in a book on layer compounds (Published by Kliewer) (DOE-ER45072-47).

3. "Charge-Density-Waves by STM", R.V. Coleman, Zhenxi Dai, W.W. McNairy, C.G. Slough and Chen Wang to be published as a chapter in a book on STM, (published by Academic Press). (DOE-ER45072-48).

\section{Papers Presented at Conference}

1. "Surface Structure of Charge-Density-Wave Materials Using Scanning Tunneling Microscopy", R.V. Coleman, Zhenxi Dai, W.W. McNairy, C.G. Slough and Chen Wang, to be presented at "First International Symposium on Atomically Controlled Surfaces and Interfaces", University of Tokyo, Tokyo Japan, Nov. 19-22, 1991. (Abstract attached).

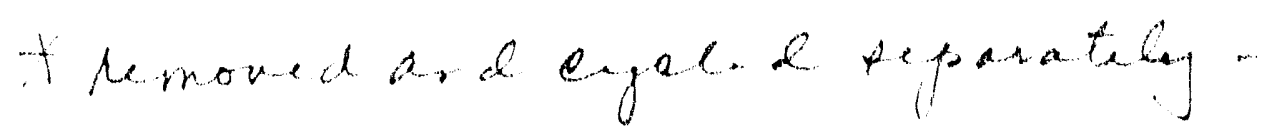




\section{Atomic Force Microscopy}

Our new Nanoscope II atomic force microscope (AFM) is assembled and running. It provides extremely high resolution of atomic and charge-density-wave (CDW) structure in transition metal dichalcognide layer compounds. The initial results were reported in publication (DOE-ER45072-40) and (DOE-ER45072-43) reprints of which are attached to this report. Work in continuing on the strong CDW materials IT- $\mathrm{TaSe}_{2}$ and IT- $\mathrm{TaS}_{2}$ with particular concentration on the CDW domain structure in IT- $\mathrm{TaS}_{2}$ at $300 \mathrm{~K}$. We have thoroughly analyzed the domain data obtained with the STM and have shown that it is consistent with the theories developed for the incommensurate domain structure in IT-TaS ${ }_{2}$. This analysis is to be published in the Physical Review B a preprint of which is attached to this report. The AFM data show a much stronger atomic modulation relative to the CDW modulation. This gives a much clearer pattern of the superposition of atoms and CDWs, but the analysis is more complex due to interference. Large area scans sufficiently well-defined to give high quality Fourier transforms are required to fully analyze the AFM results.

The AFM is being tested on a wide range of both metals and insulators. It works well on oxide surfaces and we have used it with considerable success on Fe oxide grown on a (100) single crystal surface. As shown in Fig. III-1 the oxide surface shows a characteristic pebbled surface with patches of random size. The STM scans of the same surface show an entirely different oriented structure as shown in Fig. III-2. This is due to the fact that the oxide tunnel barrier is extremely low and the STM samples the internal structure of the oxide layer rather than the surface. We expect to exploit this difference between the AFM and STM response to study all stages of the oxidation of Fe. The low barrier makes it possible to use the STM to study all stages of oxidation including very thick films. Experimental plans are outlined in the renewal proposal.

\section{Spectroscopy with the STM}

\section{A. Introduction}

We have previously demonstrated that the STM can produce high quality $\mathrm{dI} / \mathrm{dV}$ versus $\mathrm{V}$ curves from which CDW energy gaps can be measured with some precision (See publication (DOE-ER45072 
-38, Phys. Rev. B42, 8890 (1990)). We have been applying the spectroscopy techniques to dilute alloys of $\mathrm{NbSe}_{3}$ where $\mathrm{Fe}$, Co and $\mathrm{Ni}$ have been added to pure $\mathrm{NbSe}_{3}$. We have discovered very rapid changes in the CDW energy gaps and these represent both increases and decreases depending $\mathrm{n}$ the dopant. These changes in CDW gap energies may also cause changes in the CDW amplitudes in the STM scans and we are exploring this correlation.

We have previously studied ${ }^{1,2,9}$ the effects of $\mathrm{Fe}, \mathrm{Co}$ and $\mathrm{Ni}$ dopants on the quantum oscillations due to magnetic breakdown of the CDW gaps. Correlations may also exist between the STM spectroscopy and scanning results and the changes in quantum oscillation frequencies and distributions. The section below describes the initial results of the spectroscopy measurements on $\mathrm{Fe}_{0.01} \mathrm{NbSe}_{3}, \mathrm{Co}_{0.03} \mathrm{NbSe}_{3}$ and $\mathrm{Ni}_{0.03} \mathrm{NbSe}_{3}$. These are suprisingly large ef fects and we will pursue the spectroscopy measurements for wide range of dopants in $\mathrm{NbSe}_{3}$.

\section{B. Correlation of Charge-Density-Wave Energy Gaps and Impurity Pinning in $\mathrm{NbSe}_{3}$}

Measurements of the magnetoquantum oscillations in $\mathrm{NbSe}_{3}, \mathrm{Fe}_{\mathbf{x}} \mathrm{NbSe}_{3}, \mathrm{Co}_{\mathbf{x}} \mathrm{NbSe}_{3}$ and $\mathrm{Ni}_{\mathbf{x}} \mathrm{NbSe}_{3}$ have shown that the frequency and frequency distribution of the quantum oscillations are extremely sensitive to impurity pinning of the charge-density-wave (CDW). It has been argued previously ${ }^{1,2}$ that the main contribution to the ac magnetoresistance at low temperature arises from magnetic breakdown (MB) interference within a network of open and closed orbits connected by MB across the CDW gaps. Interaction between the CDW pinning impurities creates spatial variations in the CDW gaps inducing corresponding changes in the cross-sectional areas of the normal electron and hole pockets. This spatial variation of the CDW energy gaps was then identified as the mechanism for explaining the unique pattern of beats, frequencies and amplitudes observed af ter each cool-down of the crystal or after each depinning and repinning of the CDW at low temperatures.

In this paper we present data on direct measurements of the CDW gaps in $\mathrm{NbSe}_{3}$ and $\mathrm{NbSe}_{3}$ doped with $\mathrm{Fe}, \mathrm{Co}$ and $\mathrm{Ni}$ using a scanning tunneling microscope (STM). The results show that the increased pinning of the CDW due to the added impurities can substantially shift the CDW energy gaps as well as change the beat structure and frequency spread in the quantum …illations. The 
frequency ranges observed in the magnetoquantum oscillations of the doped crystals are directly correlated with the change in magnitude of the CDW energy gaps induced by the pinning impurities as measured with the STM.

For $\mathrm{NbSe}_{3}$ grown from pure starting materials the energy gaps measured at $4.2 \mathrm{~K}$ are $\Delta_{1}=35.0$ $\pm 1.5 \mathrm{meV}$ and $\Delta_{2}=101.0 \pm 1.8 \mathrm{meV}$. These values are the average of seven different measurements and agree with previous tunneling measurements of $\Delta_{1}$ by Fournel et al. ${ }^{3}$ and measurements of $\Delta_{1}$ and $\Delta_{2}$ by Ekino and Akimitsu. ${ }^{4}$ The typical structure in the $\mathrm{dl} / \mathrm{dV}$ versus $\mathrm{V}$ curves induced by the two $\mathrm{CDW}$ gaps is shown in Fig. 1(a) for pure $\mathrm{NbSe}_{3}$. The addition of dilute $\mathrm{Fe}$ impurities to $\mathrm{NbSe}_{3}$ substantially decreases the magnitude of both $\Delta_{1}$ and $\Delta_{2}$. The average values obtained from seven separate measurements are $\Delta_{1}=24.8 \pm 1.8 \mathrm{meV}$ and $\Delta_{2}=73.2 \pm 1.1 \mathrm{meV}$. A typical dI/dV versus $\mathrm{V}$ curve taken at $4.2 \mathrm{~K}$ for $\mathrm{Fe}_{0.01} \mathrm{NbSe}_{3}$ is shown in Fig. 1(b). The energy gaps associated with both CDW's have been reduced by $25-30 \%$ and the structure due to the high temperature CDW gap $\left(\Delta_{2}\right)$ is enhanced. This is a significant modification of the CDW gaps and would be expected to increase the number of normal electrons in small pockets of Fermi surface in the as cooled near equilibrium state of the CDW. This is clearly observed in the quantum oscillations of $\mathrm{Fe}_{0.01} \mathrm{NbSe}_{3}$ compared to those observed in pure $\mathrm{NbSe}_{3}$. The frequency spread required to fit the observed quantum oscillations in an as cooled $\mathrm{Fe}_{0.01} \mathrm{NbSe}_{3}$ crystal is shown in Fig. 2(b) for oscillations recorded with $\overrightarrow{\mathrm{H}}$ oriented along $\left(\overrightarrow{\mathrm{c}}+5^{\circ}\right)$. In pure $\mathrm{NbSe}_{3}$ for field directions near the $\overrightarrow{\mathrm{c}}$-axis the oscillations always show a maximum frequency of $\sim 32 \mathrm{MG}$ as confirmed in many experiments. ${ }^{1,2,6,6}$ In contrast, the as cooled $\mathrm{Fe}_{0.01} \mathrm{NbSe}_{3}$ crystals show a maximum frequency in the range 32-34 MG indicating a substantial increase in the average cross-sectional area of the Fermi surface sections governing the MB frequency. The frequency distributions required to fit the observed quantum oscillations for a particular near equilibrium state or a metastable state produced by depinning and repinning have been determined by Fourier transforms of the data or by fitting to a modified Sowa Falicov model. ${ }^{2,7}$ In both the $\mathrm{Fe}_{0.01} \mathrm{NbSe}_{3}$ and $\mathrm{NbSe}_{3}$ the frequency distribution can be narrowed and the magnitude reduced to $\sim 28 \mathrm{MG}$ by depinning and repinning the $\mathrm{CDW}$ at $1.2 \mathrm{~K}$. An example for $\mathrm{Fe}_{0.01} \mathrm{NbSe}_{3}$ is shown in Fig. 2(c) while examples for pure $\mathrm{NbSe}_{3}$ can be found in references 1 and 2 . The 
depinning process creates metastable states of the CDW in which the impurity interaction with the CDW can be substantially changed. In all cases, a depinning sequence can be found which eventually narrows the distribution and shifts it to a minimum frequency.

STM scans of the $\vec{b}-\vec{c}$ plane of $\mathrm{Fe}_{0.01} \mathrm{NbSe}_{3}$ show that all three surface chains of the unit cell still show a strong CDW modulation. A STM scan taken in the constant current mode at $4.2 \mathrm{~K}$ is shown in Fig. 3. A strong CDW modulation is observed on chains $\mathrm{I}^{\prime}$, II, and III with profiles very similar to those observed for pure $\mathrm{NbSe}_{3}$ as reported by Dai et al. ${ }^{8}$ Although the CDW energy gaps are reduced by $25-30 \%$ the Fermi surface nesting topology and wavefunction components associated with each chain must remain similar to those present in pure $\mathrm{NbSe}_{3}$.

In contrast to $\mathrm{Fe}_{0.01} \mathrm{NbSe}_{3}$, doping with Co produces the opposite effect on the CDW gaps. The average of 10 separate measurements on $\mathrm{Co}_{0.03} \mathrm{NbSe}_{3}$ at $4.2 \mathrm{~K}$ gives values of $\Delta_{1}=47.8 \pm 3.4 \mathrm{meV}$ and $\Delta_{2}=129.9 \pm 2.0 \mathrm{meV}$, values $25-30 \%$ higher than observed for pure $\mathrm{NbSe}_{3}$. A typical dI/dV versus $\mathrm{V}$ curve is shown in Fig. 4 and exhibits well defined gap structure with a substantial enhancement of the structure due to the low temperature gap $\Delta_{1}$. This increase in the magnitude of $\Delta_{1}$ is also observed to change the frequency distribution of the magnetoquantum oscillations observed in $\mathrm{Co}_{0.03} \mathrm{NbSe}_{3}$ compared to those observed in pure $\mathrm{NbSe}_{3}$.

A frequency distribution in an as cooled state for $\mathrm{Co}_{0.03} \mathrm{NbSe}_{3}$ with $\overrightarrow{\mathrm{H}} \| \overrightarrow{\mathrm{c}}$ is shown in Fig. 5(a) and shows maximum and minimum frequencies of 31.3 and $26 \mathrm{MG}$ respectively. After depinning the frequency distribution has shifted with a maximum of $29.0 \mathrm{MG}$ and a minimum of $26 \mathrm{MG}$ as shown in Fig.5(b). The maximum observed frequency in $\mathrm{Co}_{0.03} \mathrm{NbSe}_{3}$ crystals is quite variable and depends on the history of cooldowns and depinning. Generally the entire distribution is shifted to lower frequencies, but partial depinning and repinning can produce wide frequency distributions with maxima up to $35 \mathrm{MG}$ as demonstrated in Ref. 9. These higher frequency metastable states can be reduced to lower frequencies by repeated depinning and repinning of the CDW at low temperature. In all of the $\mathrm{C}_{0.03} \mathrm{NbSe}_{3}$ crystals studied the minimum frequency can be reduced to 25-26 MG after selected depinning and repinning of the CDW at low temperature. This minimum frequency is $\sim 2$ MG lower than can be observed in pure $\mathrm{NbSe}_{3}$ or in $\mathrm{Fe}_{0.01} \mathrm{NbSe}_{3}$. 
These observations of a strong downward shift in the minimum frequency in the quantum oscillations of $\mathrm{Co}_{0.03} \mathrm{NbSe}_{3}$ are consistent with the increase in the average CDW gap. Increasing the average CDW gap would be expected to condense more electrons into the CDW condensate and reduce the cross-sectional area of the normal FS which determines the frequency of the magnetoquantum oscillations.

For selected metastable states in all of the doped crystals it is possible to produce very localized pinning domains where the gap is sufficiently lowered that frequency components of $35 \mathrm{MG}$ or higher can be observed. Such local regions contribute strongly to the MB network as measured over macroscopic regions of the crystal. In contrast, STM tunneling measurements of the gap occur at an arbitrary local region a few unit cells in extent and the probability of placing the tip at a location where the gap deviates strongly from the average value is rather small. So far measurements on a given crystal at random positions all give similar gap values. Simultaneous measurement of the quantum oscillations and the tunneling spectra would be desirable since the crystal could be driven into a metastable state characterized by a narrow distribution at the lowest frequency. In this case the STM shc uld measure an enhanced CDW gap compared to the average values measured in the as cocled crystals. Experiments of this type are being developed.

In the case of $\mathrm{Ni}_{0.03} \mathrm{NbSe}_{3}$ the results differ from those observed for both $\mathrm{Fe}$ and Co doping. STM measurements of $\Delta_{1}$ and $\Delta_{2}$ are shown for a typical dI/dV versus $V$ curve in Fig. 6. The average gap values determined from 24 different measurements are $\Delta_{1}=35.6 \pm 1.5 \mathrm{meV}$ and $\Delta_{2}=116.3 \pm 2.3$ meV. The low temperature CDW gap $\Delta_{1}$, is essentially unchanged from that observed in pure $\mathrm{NbSe}_{3}$, while the high temperature CDW gap $\Delta_{2}$, is increased by $\sim 15 \%$. Since the quantum oscillations arise from $\mathrm{MB}$ of the low temperature CDW gap we do not expect the frequency distribution to shift much from that observed for pure $\mathrm{NbSe}_{3}$. In the field orientation range from $\overrightarrow{\mathrm{H}}$ parallel to $\mathrm{c}$ to $\overrightarrow{\mathrm{C}}+10^{\circ}$ the frequency distributions for $\mathrm{Ni}_{0.03} \mathrm{NbSe}_{3}$ are observed to have minimum and maximum frequencies of 28 and $32 \mathrm{MG}$ respectively, the same as observed for pure $\mathrm{NbSe}_{3}$ in this range of magnetic field orientations. (See Ref. 2). Repeated depinning of the CDW at low temperature does not lower the minimum frequency below $28 \mathrm{MG}$, the same minimum frequency as observed in pure $\mathrm{NbSe}_{3}$. 
The measurements on the $\mathrm{NbSe}_{3}$ crystals doped with $\mathrm{Fe}, \mathrm{Co}$, and $\mathrm{Ni}$ establish that the frequency range required to fit the magnetoquantum oscillations is directly related to the size of the low temperature CDW energy gap in the doped crystals. Doping the crystal with $\mathrm{Fe}$, Co or $\mathrm{Ni}$ increases the threshold electric field and enhances the number of metastable CDW states. However, the range of frequencies characterizing these metastable states is determined by the effect of the impurity on the average energy gap for the low temperature CDW. This is quite different for the three impurities tested and suggests that the detailed domain structure of the pinned CDW determines the shift of the CDW energy gap and consequently the shift of the cross-sectional area of the normal FS.

The concentrations of impurity given are those in the starting powder from which the crystal was grown. In the case of $\mathrm{Fe}$ it has been shown by secondary ion mass spectroscopy ${ }^{1}$ that the starting Fe concentration is reduced by two orders of magnitude in the final as-grown crystal. However, at starting concentrations above $\mathrm{x}=0.09$ the $\mathrm{Fe}$ is incorporated into one of the $\mathrm{Nb}$ chains and the crystal structure changes. ${ }^{10}$ The Co and Ni concentrations are also reduced in the as grown crystal, but Co and $\mathrm{Ni}$ do not induce a crystal structural change at higher starting concentrations suggesting a subtle difference in their reaction with the $\mathrm{NbSe}_{3}$ lattice compared to that of Fe. All of the doped crystals studied have RRRs in the range 30 to 120 compared to 100-300 for the pure crystals measured.

\section{References}

1. M. P. Everson, A. Johnson, Hao-An Lu, R. V. Coleman and L. M. Falicov, Phys. Rev. B $\underline{36}, 6953$ (1987).

2. R. V. Coleman, M. P. Everson, Hao-An Lu, A. Johnson and L. M. Falicov, Phys. Rev. B 41,460 (1990).

3. A. Fournel, J. P. Sorbier, M. Konczykowski, and P. Monceau, Phys. Rev. Lett. $\underline{57}, 2199$ (1986).

4. T. Ekino and J. Akimitsu, Jpn. J. Appl. Phys. 26-3, 625 (1987).

5. R. V. Coleman, M. P. Everson, G. Eiserman, and A. Johnson, Physica 143B, 33 (1986).

6. R. V. Coleman, M. P. Everson, L. M. Falicov, Hao-An Lu, and A. Johnson, Jap. J. Appl. Phys. $\underline{26}, 615-616(1987)$.

7. E. C. Sowa and L. M. Falicov, Phys. Rev. B $\underline{32,} 755$ (1985). 
8. Zhenxi Dai, C. G. Slough and R. V. Coleman, Phys. Rev. Lett. $\underline{66}, 1318$ (1991).

9. Hao-An Lu, C. G. Slough, R. V. Coleman, Amitesh Maiti and L. M. Falicov, Phys. Rev. B444, (1991).

10. S. J. Hillenius, R. V. Coleman, R. M. Fleming and R. J. Cava, Phys. Rev. B 44 , (1981). 


\author{
Abstract \\ First International Conference on Atomically Controlled \\ Surfaces and Interfaces \\ Surface Structure of Charge-Density-Wave Materials \\ Using Scanning Tunneling Microscopy \\ by \\ R. V.Coleman, Zhenxi Dai, W. W. McNairy \\ C. G. Slough and Chen Wang \\ Department of Physics \\ Univarsity of Virginia \\ Charlottesville, VA 22901
}

The scanning tunneling microscope (STM) not only gives excellent atomic resolution of surfaces, but also responds to the detailed electronic structure of the material. Charge-density-waves (CDWs) represent an instability of the Fermi surface leading to the formation of standing waves, modif ications in the local density-of-states and in some cases unusual conduction properties. We present STM data on a wide range of the CDW materials exhibiting several different types of CDW structure. ${ }^{1}$ The materials include the quasi-two-dimensional crystals $\mathrm{TaS}_{2}, \mathrm{TaSe}_{2}$ and $\mathrm{NbSe}_{2}$ and the quasi-onedimensional materials $\mathrm{NbSe}_{3}$ and $\mathrm{TaS}_{9}$. CDW phases are observed that are both commensurate and incommensurate with the lattice. In several materials and phases two separate CDW transitions are observed. The STM can detect the relative CDW amplitudes and charge distributions as well as wavelengths, domain structures and positions with respect to the atomic lattice. In the case of the linear chair laterials, $\mathrm{NbSe}_{3}$ and $\mathrm{TaS}_{3}$, detailed identification of the specific chains carrying the $\mathrm{CDW}$ modulation has been obtained. $\mathrm{CDW}$ modulation due to the low temperature $\mathrm{CDW}$ in $\mathrm{NbSe}_{3}$ has been observed ${ }^{2}$ to exist on two pairs of chains rather than on just one pair of chains. For the layer structure crystals such as $\mathrm{TaS}_{2}$, detailed CDW domain structure in the nearly commensurate phase of $1 \mathrm{~T}-\mathrm{TaS}_{2}$ has been obtained along with complete information on the commensurate phase. The amplitude modulation of the CDWs in the nearly commensurate phase has been measured and is in close agreement with theory. In the mixed coordination phases $4 \mathrm{Hb}-\mathrm{TaS}_{2}$ and $4 \mathrm{Hb}-\mathrm{TaSe}_{2}$ the two 
independent CDWs can be simultaneously detected. The spectroscopic mode of the STM has also been used to measure the CDW energy gaps in all of the CDW materials studied by STM. Two CDW gaps have been resolved in those materials exhibiting two CDW transitions. All of the CDW gaps values indicate strong coupling with the strongest coupling observed for the $2 \mathrm{H}$ phase compounds. The STM results on CDW structure demonstrate the sensitive response of the STM to modifications of the electronic structure near the Fermi level.

1. R. V. Coleman, B. Giambatista, P. K. Hansma, A. Johnson, W. W. McNairy, and C. G. Slough, Adv. in Phys. $\underline{37}$, 559-644 (1988).

2. Zhenxi Dai, C. G. Slough and R. V. Coleman, Phys. Rev, Lett. $\underline{66}, 1318$ (1991). 
STM Fe (100)

High Temperature Oxidation

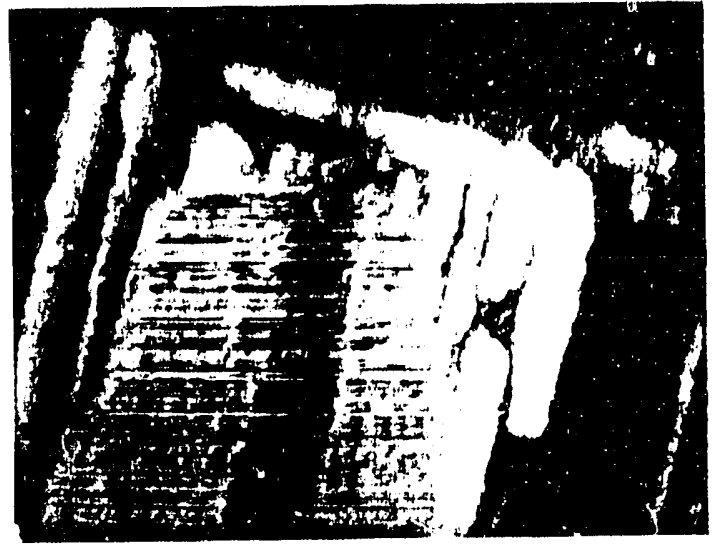

(a)

$$
\begin{aligned}
& \text { Scan Size }=13 \mathrm{~km} \\
& Z-\text { Range }=450 \mathrm{~nm} \\
& 450^{\circ} \mathrm{C} \text { Oxidation }
\end{aligned}
$$

(b)

$$
\begin{aligned}
& S \text { can Size }=13.2 \mu \mathrm{m} \\
& z-\text { Range }=0.4 \mu \mathrm{m}
\end{aligned}
$$$$
350^{\circ} \mathrm{C} \text { Oxidation }
$$
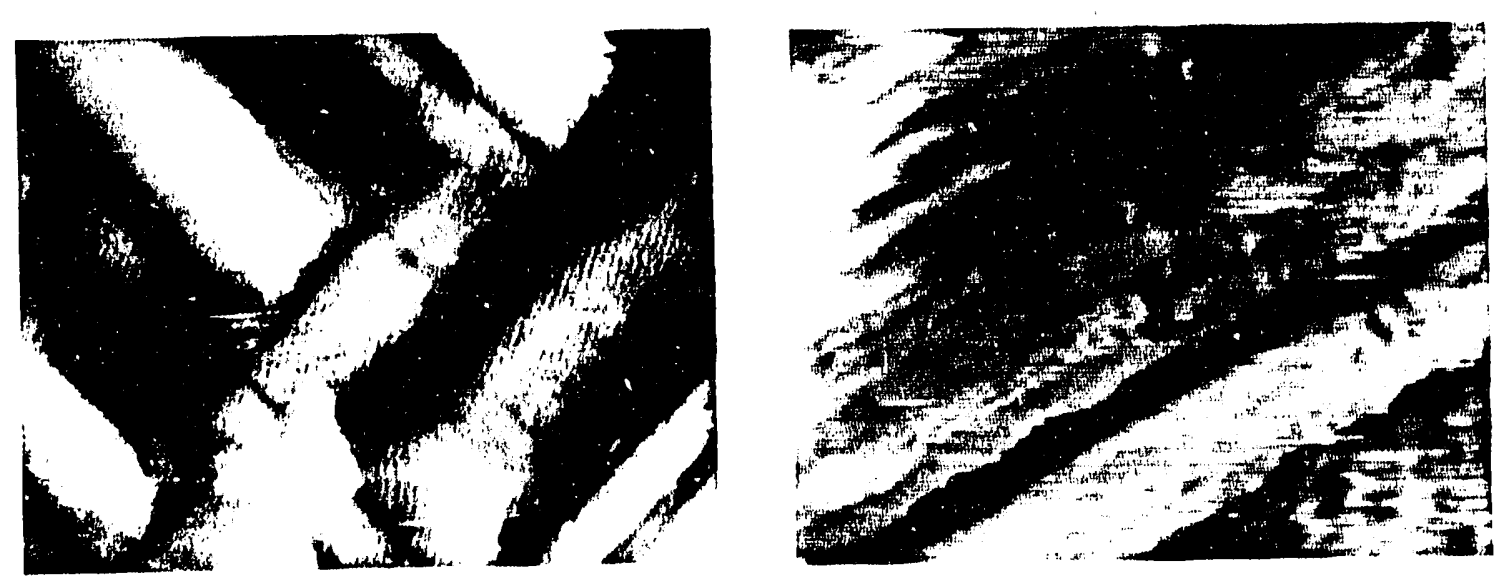

(c)

$$
\begin{aligned}
& \text { Scan Size }=13 \mu \mathrm{m} \\
& Z-\text { Range }=350 \mathrm{~nm} \\
& 400^{\circ} \mathrm{C} \text { Oxidation }
\end{aligned}
$$

(d)

$$
\begin{aligned}
& \text { Scan Size }=580 \mathrm{~nm} \\
& Z-\text { Range }=70 \mathrm{~nm} \\
& 350^{\circ} \mathrm{C} \text { Oxidation }
\end{aligned}
$$




$$
\text { AFM Fe }(100)
$$

High Temperature Oxidation

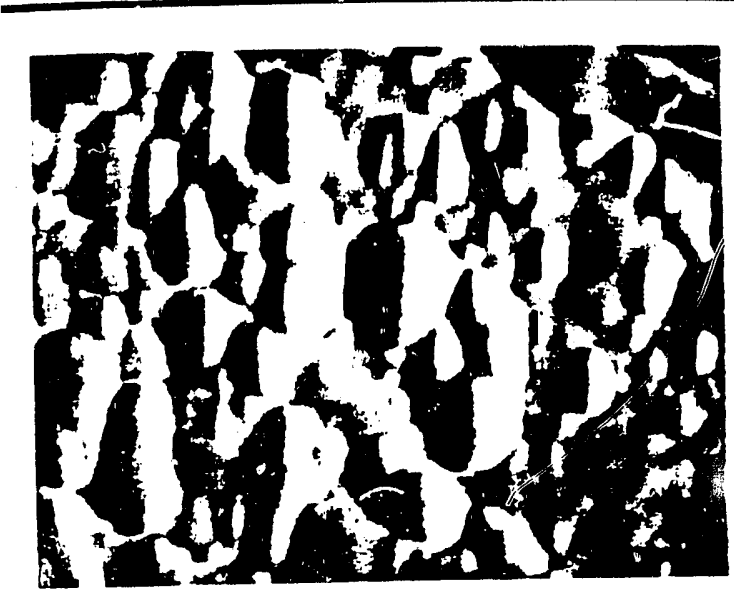

(a)

$$
\begin{aligned}
& \text { Scan Size }=1000 \mathrm{~nm} \\
& Z-\text { Range }=50 \mathrm{~nm} \\
& 400^{\circ} \mathrm{C} \text { Oxidation }
\end{aligned}
$$

(b)

$$
\begin{aligned}
& \text { Scan Size }=1.025 \mu \mathrm{m} \\
& Z-\text { Range }=30 \mathrm{~nm} \\
& 450^{\circ} \mathrm{C} \text { Oxidation }
\end{aligned}
$$

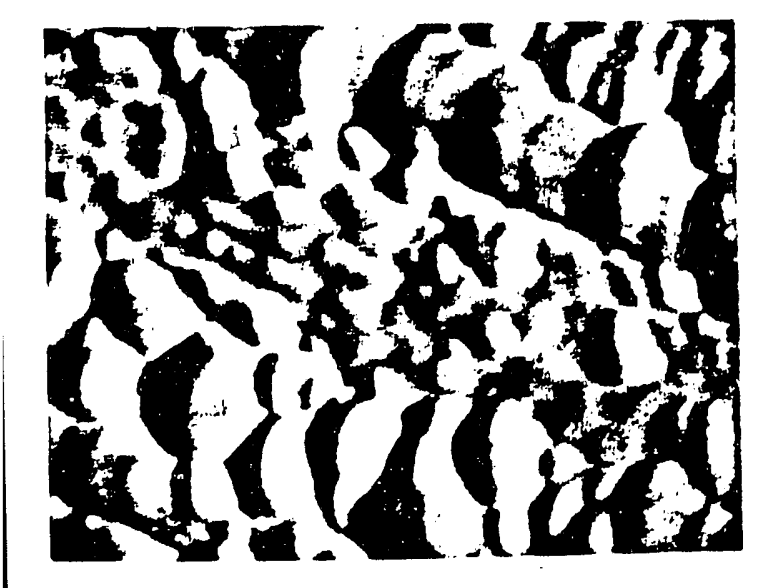

(c)

$$
\begin{aligned}
& \text { Scan Size }=1000 \mathrm{~nm} \\
& \text { z-Range }=50 \mathrm{~nm} \\
& 450^{\circ} \mathrm{C} \text { Oxidation }
\end{aligned}
$$

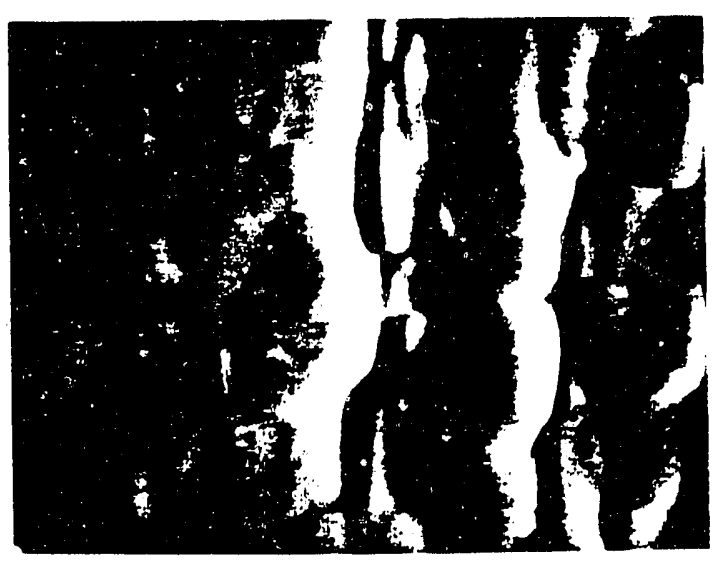

(d)

$$
\begin{aligned}
& \text { Scan Size }=800 \mathrm{~nm} \\
& Z-\text { Range }=35 \mathrm{~nm} \\
& 450^{\circ} \mathrm{C} \text { Oxidation }
\end{aligned}
$$




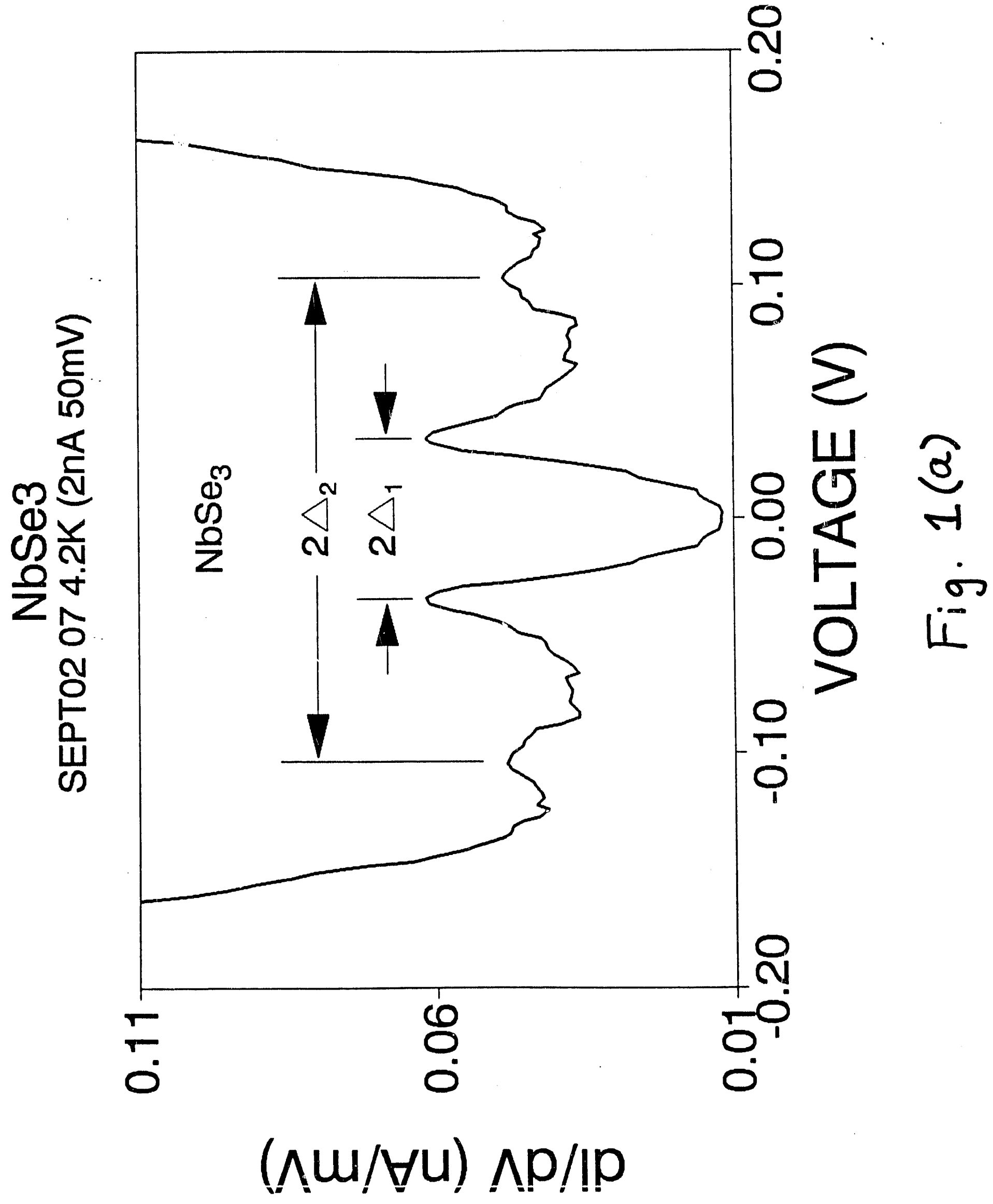




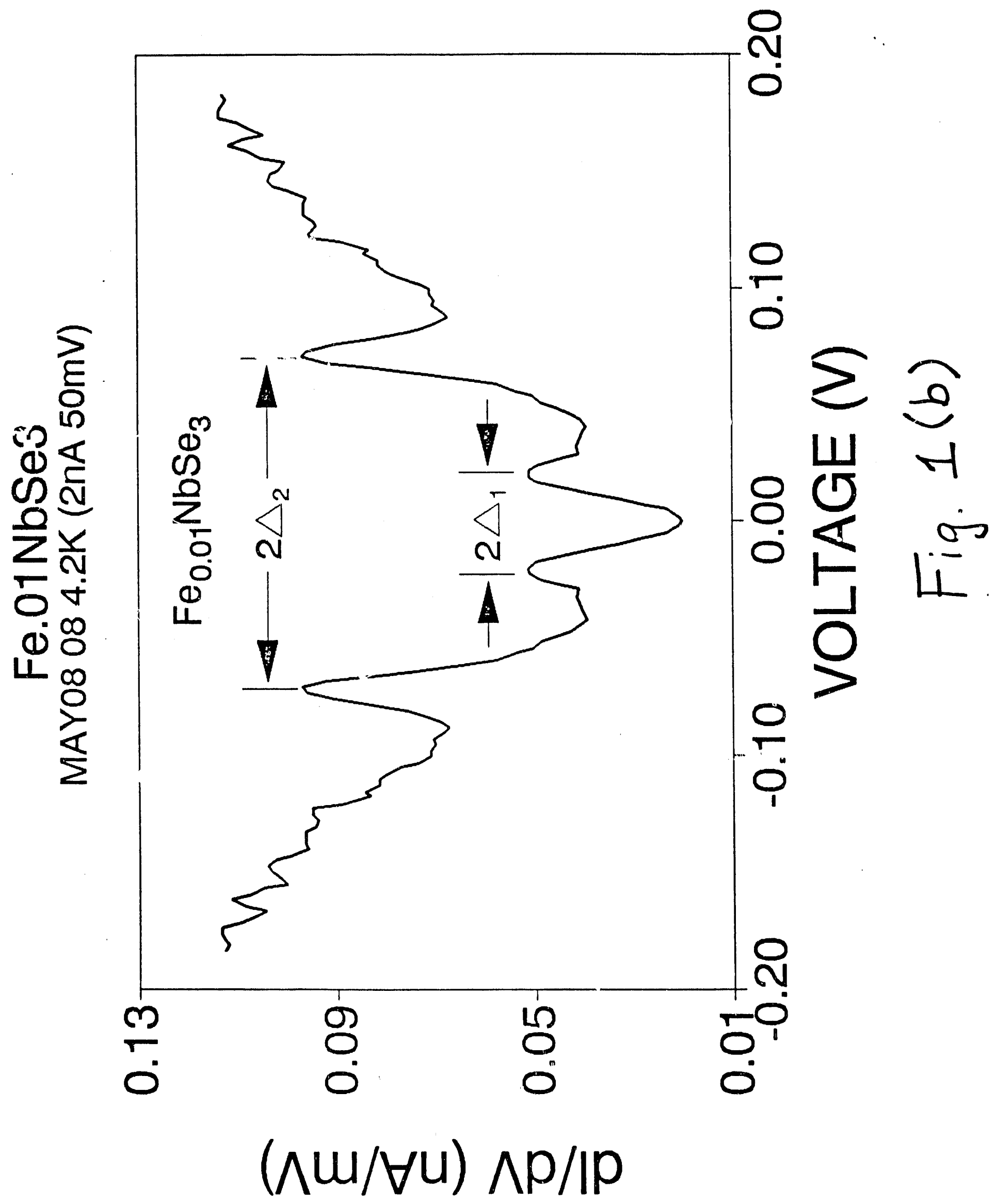




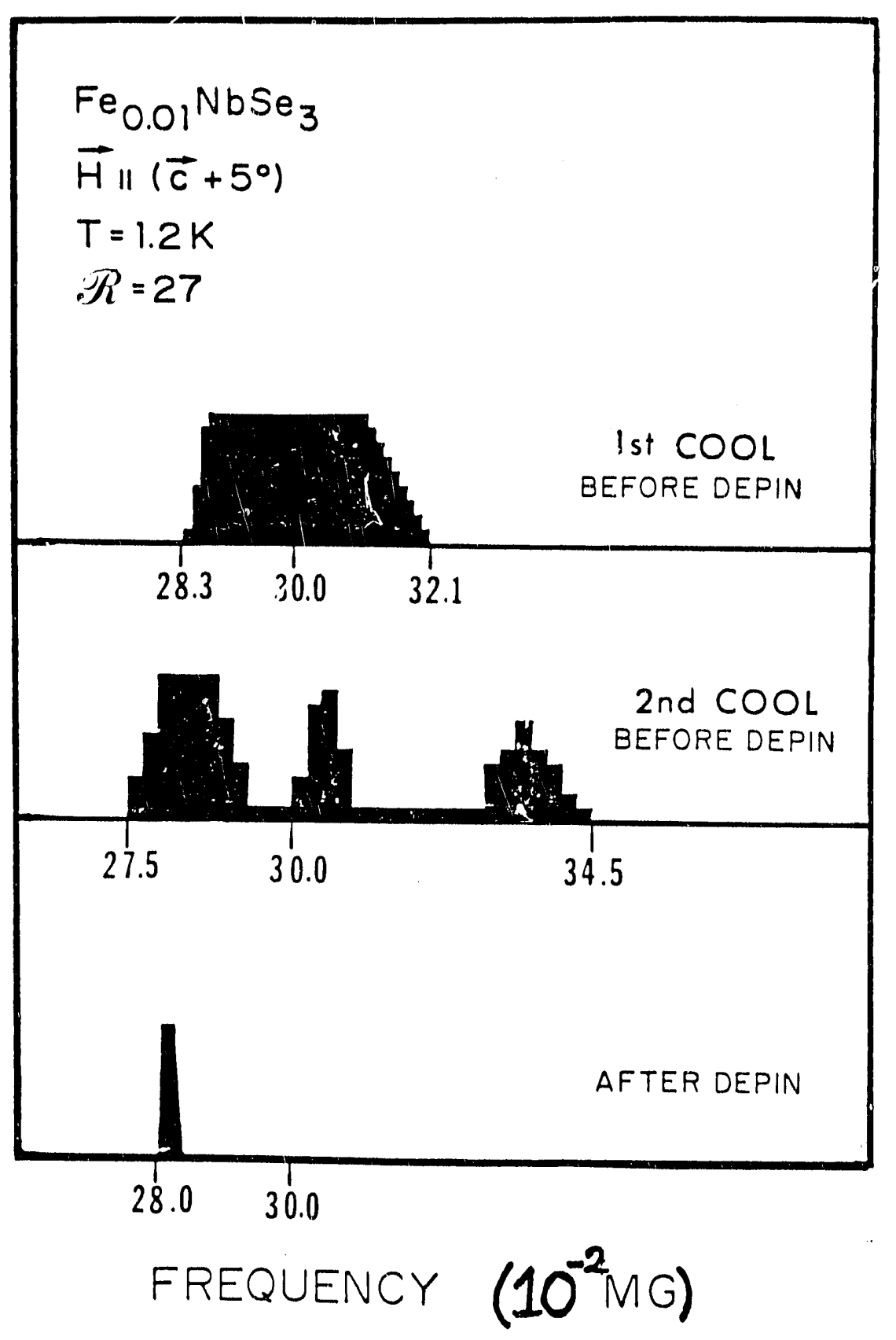

$$
\text { Fig. } 2
$$




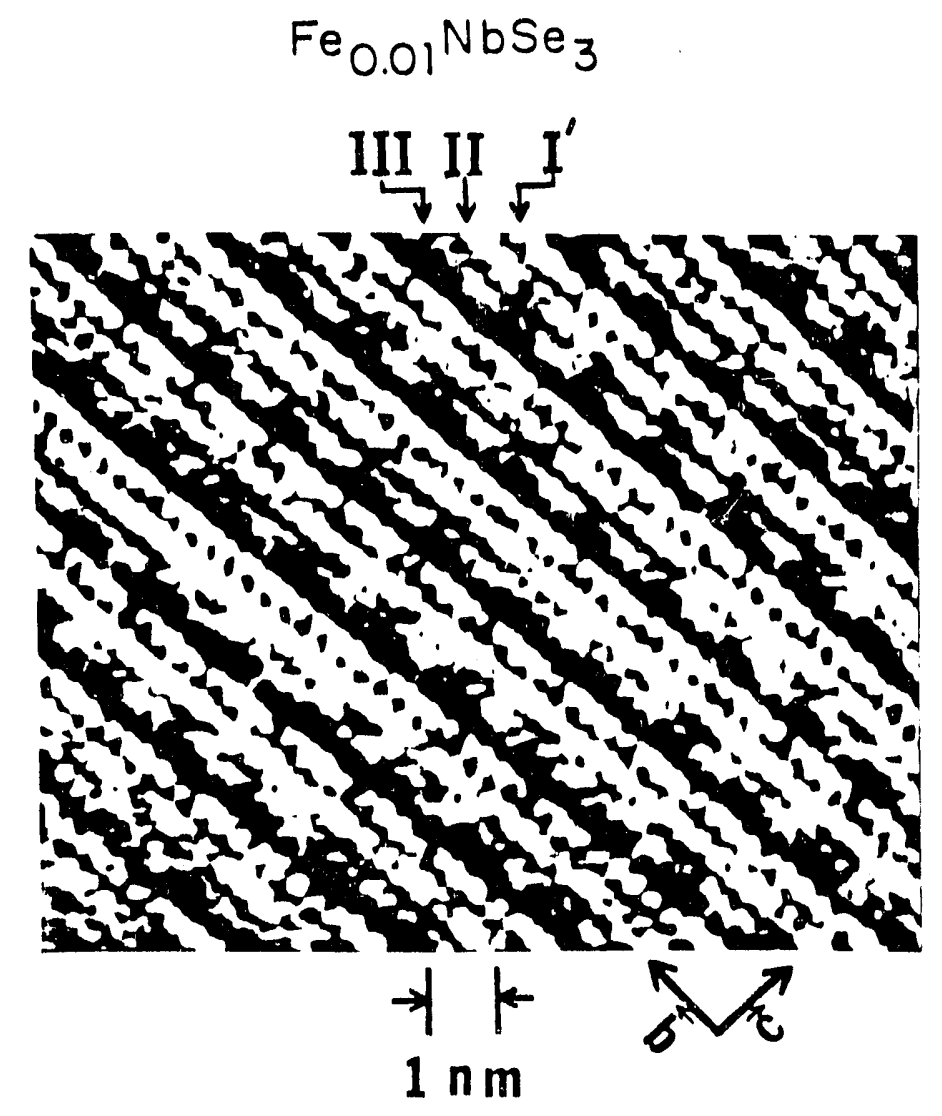

Fig. 3 


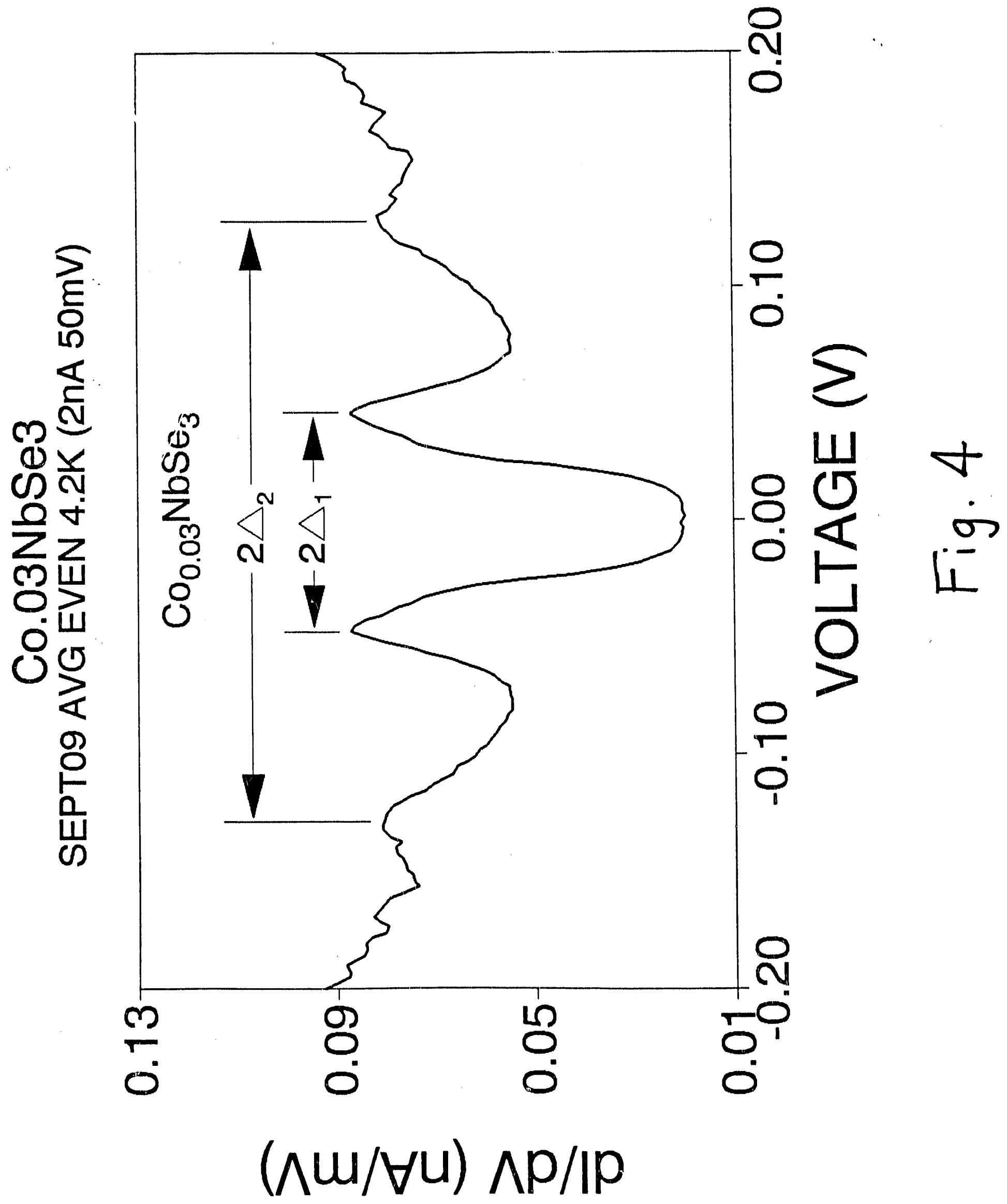




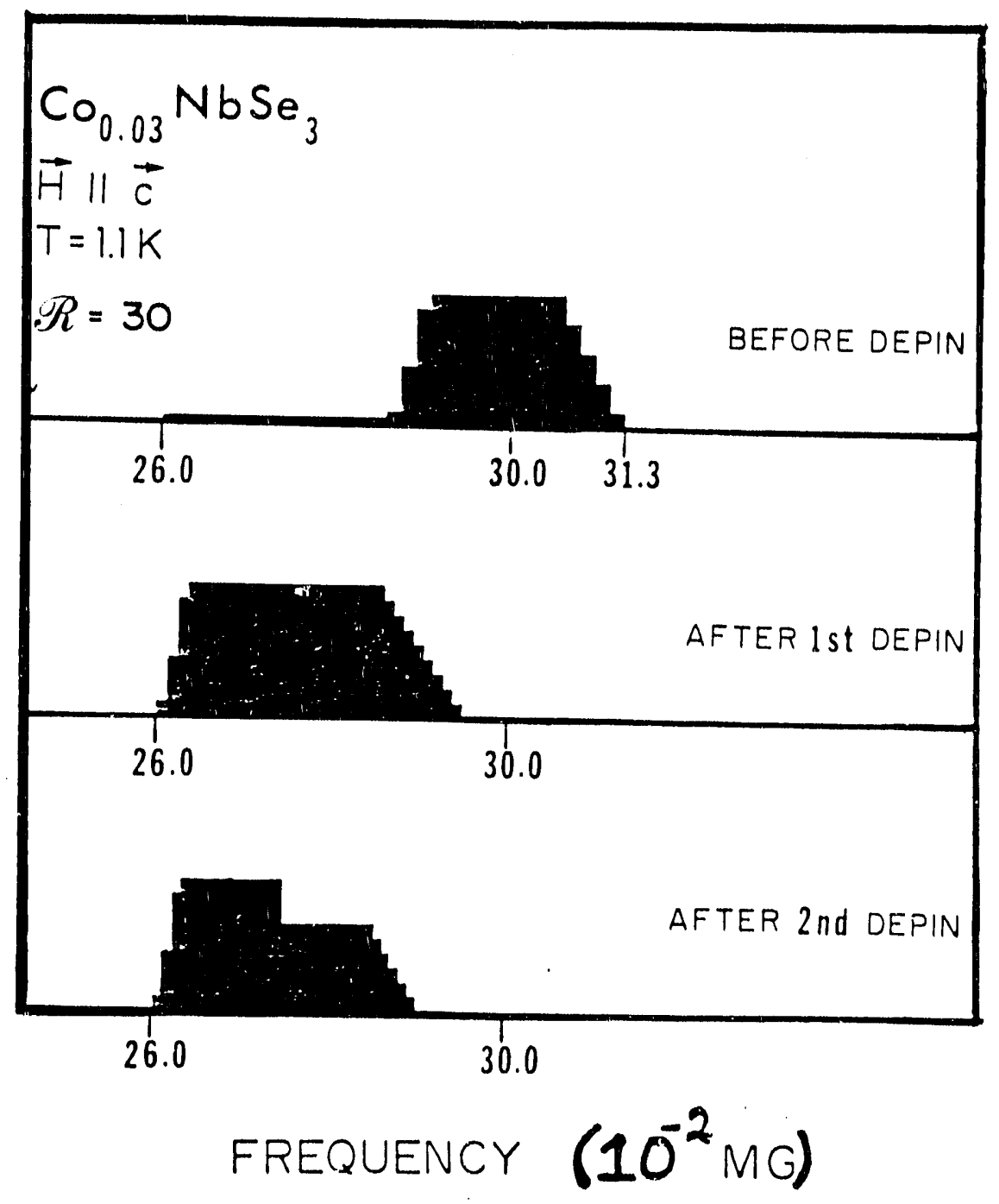

Fig. 5 


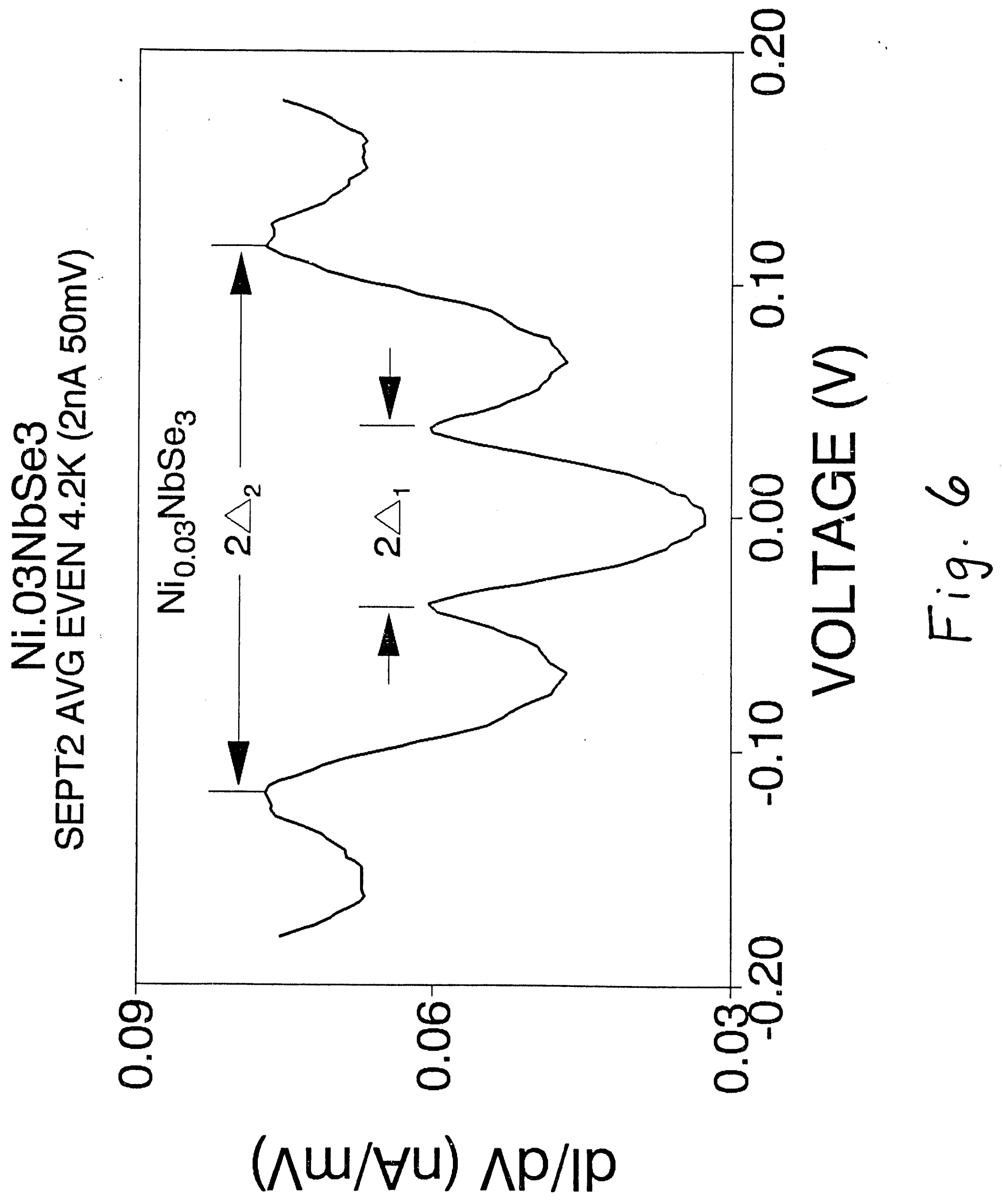



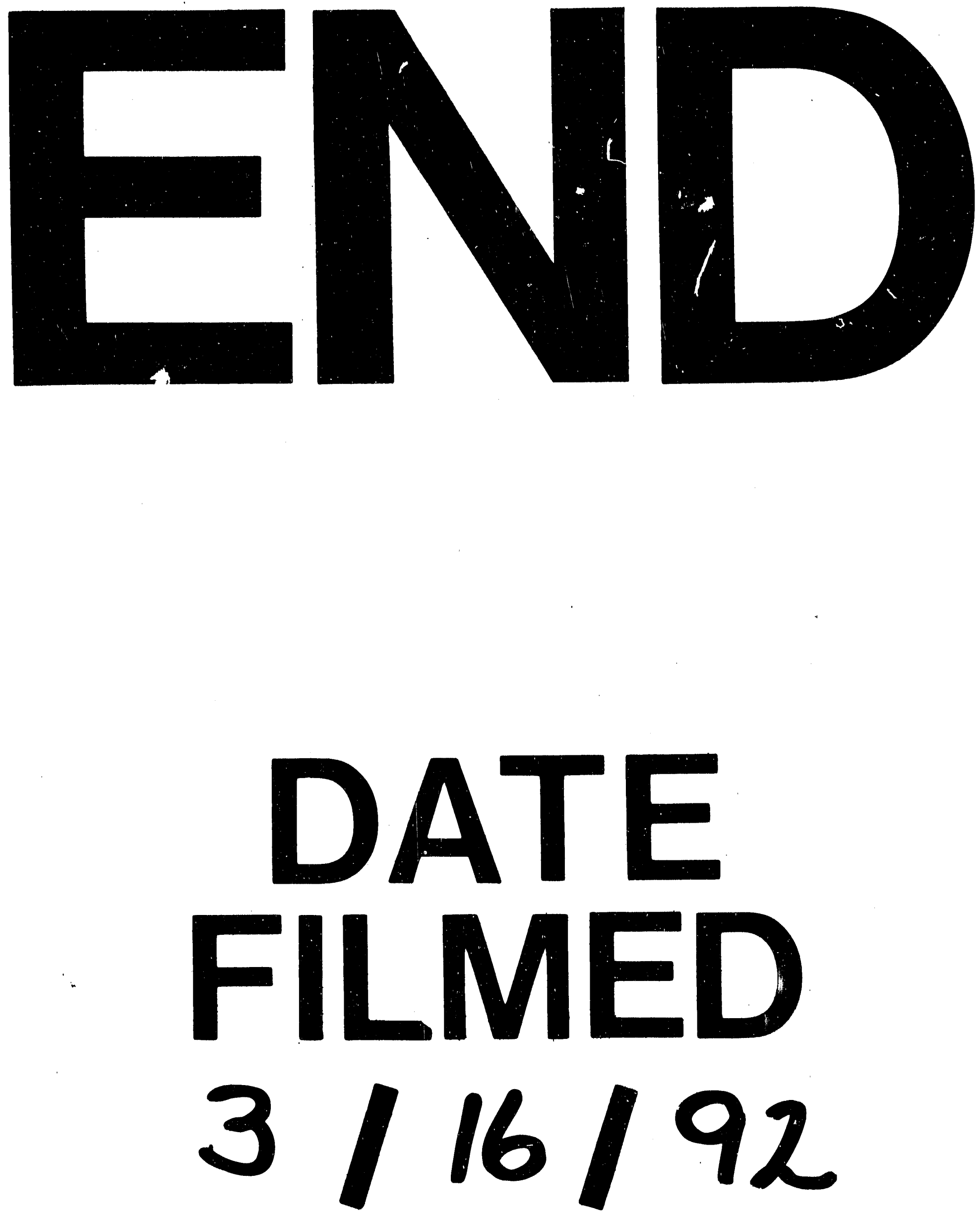
n 